\title{
Online indexing and clustering of social media data for emergency management
}

\author{
Daniela Pohl ${ }^{\mathrm{a}, *}$, Abdelhamid Bouchachia ${ }^{\mathrm{b}}$, Hermann Hellwagner ${ }^{\mathrm{a}}$ \\ a Institute of Information Technology, Alpen-Adria-Universität Klagenfurt, Universitätsstr. 65-67, Klagenfurt, Austria \\ ${ }^{\mathrm{b}}$ Smart Technology Research Center, Bournemouth University, Poole House Talbot Campus, Fern Barrow Poole, BH12 5BB Bournemouth, UK
}

\section{A R T I C L E I N F O}

\section{Article history:}

Received 28 November 2013

Received in revised form

13 January 2015

Accepted 26 January 2015

Keywords:

Event detection

Information filtering

Online indexing

Online clustering

Emergency management

\begin{abstract}
A B S T R A C T
Social media becomes a vital part in our daily communication practice, creating a huge amount of data and covering different real-world situations. Currently, there is a tendency in making use of social media during emergency management and response. Most of this effort is performed by a huge number of volunteers browsing through social media data and preparing maps that can be used by professional first responders. Automatic analysis approaches are needed to directly support the response teams in monitoring and also understanding the evolution of facts in social media during an emergency situation. In this paper, we investigate the problem of real-time sub-events identification in social media data (i.e., Twitter, Flickr and YouTube) during emergencies. A processing framework is presented serving to generate situational reports/summaries from social media data. This framework relies in particular on online indexing and online clustering of media data streams. Online indexing aims at tracking the relevant vocabulary to capture the evolution of sub-events over time. Online clustering, on the other hand, is used to detect and update the set of sub-events using the indices built during online indexing. To evaluate the framework, social media data related to Hurricane Sandy 2012 was collected and used in a series of experiments. In particular some online indexing methods have been tested against a proposed method to show their suitability. Moreover, the quality of online clustering has been studied using standard clustering indices. Overall the framework provides a great opportunity for supporting emergency responders as demonstrated in real-world emergency exercises.
\end{abstract}

(c) 2015 Elsevier B.V. All rights reserved.

\section{Introduction}

Access to information is fundamental during emergency management in order to deal efficiently with different sorts of incidents (e.g., traffic accidents, hurricanes, earthquakes, terror attacks). Collecting this information is not always an easy task, especially when relief units are not immediately on-site, e.g., due to the distance or street damages. Social media (e.g., Twitter) offers a new opportunity for supporting emergency management by enabling collection of data.

Studies $[1,2]$ show the potential of social media in different emergency situations. People report on any kind of emergency situation they witness. Therefore, social media has become an important instrument to exchange information, thus providing additional perspectives on emergency situations [3].

\footnotetext{
* Corresponding author. Tel.: +434632700 3688; fax: +434632700993688.

E-mail addresses: daniela@itec.uni-klu.ac.at (D. Pohl), abouchachia@bournemouth.ac.uk (A. Bouchachia), hellwagn@itec.uni-klu.ac.at (H. Hellwagner).
}

However, intelligent analysis methods are needed to relieve emergency responders from a cumbersome manual browsing task through this data, which is potentially noisy. Methods should be able to summarize the ongoing situation and provide an overview of the emergency situation at hand. In this paper, we focus on the detection of sub-events, i.e., specific crisis-related hotspots (e.g., flooding in a specific district of a city, power outage in another district) that emergency personnel should be aware of when organizing their intervention.

In our early work [4], we examined clustering algorithms for their suitability to detect sub-events from social media. We used Flickr and YouTube data for aftermath analysis of the crisis situation. In particular our investigations relied on offline clustering which is inappropriate for real-time analysis during the emergency situation.

We introduced an online sub-event detection mechanism [5] which combines real-time clustering and online indexing (i.e., weighting and selection of indexing terms). The sub-events (clusters) are detected and tracked as new items from social media users become available. In [5], the mechanism is used to analyze data from the Hurricane Sandy 2012 in the form of batches. It handles data 
collections from Twitter, Flickr and YouTube. We extract terms as features from the textual metadata of the incoming items. We do not process videos from YouTube and images from Flickr and do not analyze their contents, we rather extract their textual metadata (title, description, and tags) to be used along with tweets. Initial experiments on this data show the suitability for detecting topics related to the crisis at hand.

We integrated our online detection mechanism in a media exploration framework. For evaluation of the online processing method, we implement similar indexing methods and compare them with our indexing approach. Hence, the focus of this paper is on the examination of the indexing methods. In doing so, we adapted the online clustering algorithm described in [6] to meet the context of our present application. The experimental setting and the results regarding the different methods are described. They emphasize the suitability of our idea of online indexing for processing social media data.

The structure of the paper is as follows. Section 2 discusses the related work. Section 3 addresses the terminology, i.e., difference between events and sub-events, and highlights it in the context of topic detection and tracking. Section 4 introduces our suggested "Multimedia Exploration Framework". Section 5 outlines the online sub-event detection, especially the interrelationship between online indexing and clustering algorithms. Section 6 describes the details of the online indexing (i.e., implementations and our learning and forgetting model). Section 7 depicts the used online clustering algorithm in this context. In Section 8, the experimental setting and the results are presented. Section 9 concludes the paper.

\section{Related work}

The present work is related to "topic detection and tracking" in the area of social media and to "indexing and feature selection" methods.

\subsection{Topic detection and tracking}

In fact, Twitter is very popular in social media analysis and detection. For example, Gao et al. [7] present an approach that colors geographical regions (social pictures) based on their importance for the topic of interests given by the messages related to these areas. The aggregation and coloring are based on a predefined algebra. The algebra also allows the combination of different social pictures (i.e., with multimedia processing like convolution or segmentation).

Lampos and Cristianini [8] identify important keywords from auxiliary sources, e.g., Wikipedia. These keywords are searched in tweets and scored according to the amount of keywords in the tweet (e.g., to identify the daily flu-rate based on incoming tweets) [9].

Krstajic et al. [10] show an event detection mechanism based on different scores that are calculated and combined by the preferences of the user. First, terms are extracted and combined to episodes (i.e., sets of tweets). After a predefined number of tweets shown to the system, the scores are calculated. If the combined score reaches a threshold, the episode is shown to the user as a new event.

Chakrabarti et al. [11] describe a detection mechanism based on initially learned terms and their importance for a specific event (e.g., football game). In contrast, Shen et al. [12] base their detection on general concepts (e.g., name of companies or persons). General concepts are aggregated together based on their contextual and lexical similarity. Tweets depending on the resulting bag-of-words clusters are analyzed via spike detection and shown to the user. Marcus et al. [13] summarize or identify events based on the peak detection mechanism (covering a one-minute time window). Klein et al. [14] analyze tweets in real time for emergency management. They introduce a graph analysis approach. It allows them to identify leading writing users as the origin of the information spreading. Cataldi et al. [15] describe in their work also a topic detection mechanism for Twitter considering the relation between users, i.e., followers. However, in emergencies people can write about the same events although there is no relation given between them.

Allan et al. [16] describe an approach for detecting and tracking specific events. Nallapati et al. [17] use agglomerative clustering to identify events in a static manner. Osborne et al. [18] describe an online story detection mechanism based on Twitter which uses Wikipedia to verify the identified stories. The framework described by O'Connor et al. [19] analyzes previously fetched tweets to identify and summarize topics. Starbird [20] introduces Tweak-the-Tweet, which defines and uses a predefined grammar for tweets to analyze them accordingly. Twitcident, by Abel et al. [21], is based on predefined keywords or manually inserted rules. CrisisTracker by Rogstadius et al. [22] (based on [13]) represents a crowdsourcing tool to support volunteers in processing of messages coming from the public during a crisis. It uses an initial term frequency-inverse document frequency (tf-idf) model based on a sample set of tweets.

Most of the approaches use additional or auxiliary material for detection, e.g., Wikipedia, previously processed training sets, or are based on a static analysis. Most of them (e.g., Wikipedia entries or a training set) are often not available during emergencies, especially in fast evolving scenarios.

\subsection{Topic detection and tracking based on visual items}

In addition to microblogs and text messages, visual items are important in the context of crisis management. Visual items (e.g., pictures and videos) give additional insights into the incident. For example, Chen and Roy [23] perform event detection based on tags annotating Flickr images. The approach allows them to identify periodic and non-periodic events. The tags are examined based on their temporal and spatial distribution and aggregated if they are similar (i.e., representing the same event). The approach allows them also to uncover the time and location of an event. In [24] an approach is proposed to identify disaster events from Flickr. It identifies bursty tags in a predefined time interval and fetches images related to a predefined number of tags. Rattenbury et al. [25] make also use of tags to identify events from Flickr. The identification process is based on a clustering algorithm that takes into account the distribution of tags over time. It is based on specific intra and inter-cluster relationship metrics to identify event-related clusters.

Another approach from Liu et al. [26] identifies events in Flickr images based on the number of items per day coming from unique users. If the number of the incoming items is above the median, a new event is declared. Petkos et al. [27] identify events from Flickr images/items based on Support Vector Machines. Support Vector Machines are proposed to decide/classify if two items belong to the same event. A graph representation is created, where nodes represent items and edges indicate if two items belong to the same event based on the decision of the Support Vector Machines. Community detection algorithms are applied to assign items to events. Rabbath et al. [28] investigate event detection from Facebook by locating photos of the same event shared by friends.

These studies use tags associated with images and were sometimes combined with visual features extracted from the images/ videos (e.g., $[28,27])$ to detect events from social media. In a step forward, we rather use microblog texts in addition to textual annotations of the images and videos. 


\subsection{Event detection and clustering in image/video data}

Analysis approaches used for event detection in image/video streams are specific to the visual nature of the data. There, the visual content of images is used which differs from that used in the social media context of this work.

For example, Zha et al. [29] deal with the detection of events from video streams, e.g., received from surveillance cameras. The authors examine surveillance videos, e.g., from a building in a university. In this context, events are specific group activities, like queueing, discussing, joining, leaving, etc. The approach is based on a new feature called vigilant area (VA) and a graphical model [29]. The VA feature is independent from object tracking conditions and is based on space and time information considering also shape and vigilance [29]. The approach introduced by Ke et al. [30] allows them to identify specific events from videos (i.e., waving with arms or picking up something from the ground). It can detect those events from videos with crowded and cluttered background. The approach covers a shape-based matching algorithm operating in time and space dimensions of the examined video [30]. Schüldt et al. [31] discuss an approach to identify specific actions of people in videos (e.g., running, walking, and boxing) using several spatialtemporal features. Support Vector Machines are trained with these features to recognize the actions within new videos. In addition, a survey on activity detection summarizing different features and recognition approaches can be found in Aggarwal and Ryoo [32].

Image clustering approaches are used for several purposes in research. For example, Zha et al. [33] introduce an image search engine which combines text and content-based image analysis to find images of interest (e.g., apple). First, the system identifies additional related keywords (e.g., fruit and mobile phone) from an existing platform (i.e., Flickr) to refine the user search query. Second, for each identified keyword, the system suggests exemplified images to improve the results of the query. The system combines the Affinity Propagation and the k-mean clustering approach to return images that match to the meaning of the keyword [33]. Chen et al. [34] use image clustering to structure the result list of image search. The idea is to cluster semantically related images by using the $\mathrm{k}$ Nearest-Neighbor approach. Hence, a list of image clusters is shown to the user as result. Papagiannopoulou and Mezaris [35] apply clustering for image collection summarization. The authors test different visual features (e.g., SIFT) and clustering algorithms (e.g., k-means algorithm). In addition, Jaffe et al. [36] describe a summarization approach of large image collections based on hierarchical clustering. The authors use different features for clustering (e.g., geo-information and textual information).

Event detection is popular in various applications and uses different techniques such as clustering for surveillance monitoring and repository summarization.

\subsection{Indexing, weighting and feature selection}

Processing of natural language (e.g., tweets) results in a huge number of indexing terms/features. Feature selection mechanisms are used to deal with such a big feature space. In clustering, feature selection is based on the inherent structure of the data (e.g., frequency metrics) as there are no labels. Witten et al. [37] describe in the context of the WEKA framework [38] several feature selection mechanisms based also on the intrinsic data characteristics.

Yang and Pedersen [39] analyze different metrics for clustering (e.g., document frequency, mutual information, and information gain). Liu et al. [40] describe feature selection for clustering based on an Expectation Maximization (EM) algorithm. Additionally, the work by Liu and Yu [41] describes such feature selection mechanisms for clustering. Also, the works in [42] and [43] cover feature selection in the clustering context. Ahmed and Xing [44] extend the Hierarchical Dirichlet Process for describing the evolution of topics and their related words. In addition, Alelyani et al. [45] give an overview on feature selection for clustering.

Brants et al. [46] describe the creation of an index via incremental term frequency-inverse document frequency (tf-idf). Also Marcus et al. [13] make use of the incremental tf-idf. Khy et al. [47] use tf-idf in the context of clustering aging documents. Lee et al. [48] include a statistical function (i.e., skewness) to identify "bursty keywords" in the context of tweets. Also, Lampos et al. [8] make use of such important and frequent keywords for tweeting analysis. Shen et al. [12] identify important terms (e.g., representing specific concepts like names of persons and organizations) for clustering information. Devaney and Ram [49] show a clustering system (i.e., COBWEB) that guides the feature selection process. Singhal et al. [50] show an approach for pivoted tf-idf in the context of profile learning.

Also, a weighting mechanism can be used to perform feature selection or indexing, which allows the modeling of changing topics in streaming data. These weighting mechanisms are mainly coming from the classification area (i.e., labeled data forms the base for the selection process). Bouchachia and Mittermeir [51] describe a feature weighting mechanism for fuzzy classification. Wu et al. [52] show a feature selection method for streaming applications. Relevant features are selected using conditional independency. Guan and Li [53] show incremental features based on changing the topology of the underlying neural network. Rückstieß et al. [54] show an online feature selection in the context of classification. Also, Katakis et al. [55] describe an approach for dealing with concept drift in text-based data streams. In the classification context, they use labeled data and classto-term statistics. However, this information is not known in emergency management applications since the exact classes (i.e., incidents) are usually not known in advance.

In this contribution, we focus on clustering methods, which do not need labeled data or additional effort before starting the analysis (e.g., training period). Additionally, we consider a weighting mechanism for a smooth feature selection and reduction. Our goal is to identify important sub-events, i.e., hotspots of the crisis, in an online manner and group new incoming data according to these sub-events for emergency management support. In this contribution, we compare our weighting approach with two others adapted for our needs (see Sections 7.1 and 7.2).

\section{Terminology}

An event (i.e., the crisis itself) is described by its time and location (e.g., Hurricane Sandy in the USA in 2012). It is defined as follows:

Definition 1. An event is a real-world crisis related to a specific time and location.

In general, events can be seen as a composition of smaller parts, called sub-events. Sub-events correspond to different incidents of a crisis, e.g., flooding, damages, and power outage. Sub-events originate in the context of the parent event showing specific hotspots or incidents on a smaller scale, both in space and in time, e.g., flooding in Lower Manhattan NY. A sub-event summarizes postings covering similar incidents several people are writing about. A sub-event is defined as follows:

Definition 2. A sub-event is a specific incident that originates in the context of an event (the crisis).

A sub-event can be identified by incoming information describing the same specific incident, e.g., reports, tweets, pictures, and videos. Sub-event detection can indeed be seen as event detection, as discussed in Section 2. However, in our work we prefer to define 
"event" as the major happening (e.g., "Hurricane Sandy") which is known to us. Sub-events are those hotspots that collectively form the event. In other words, a sub-event is something that occurs in a certain place, as part of an event, during a particular interval of time. Therefore, we do know the event, but we do not know those hotspots, hence it is important to detect sub-events automatically. Our aim is to uncover sub-events that are triggered by a parent event using social media. The idea is similar to "topic detection and tracking" (TDT) [17], where a topic consists of different events (sub-topics) that are triggered by the topic. We use "events" and "sub-events" as a more specific emergency-related terminology.

We also have a specific spatial-temporal focus on the data itself. A data item used in our identification process consists of geoinformation (i.e., longitude and latitude) and textual data of the incoming items (e.g., the tweet itself or metadata annotations related to a picture or video). Like with TDT, we have a fast evolving situation depending on the nature of the crisis. In addition, it is very difficult to label data items in real time due to the nature of an emergency.

\section{Multimedia exploration framework}

In [4] we suggested a Multimedia Exploration Framework (MEF) for social media analysis in emergency management. We extended the framework from [4] with additional functionalities (see Fig. 1) including online analysis, geo-tagging, and an interactive visualization of the results.

The current version of the MEF now enables both online analysis and offline analysis of social media data. The streaming interface allows data to be fetched in real time. Additionally, we included a geo-tagging mechanism which can be used to automatically tag incoming information. This is performed via named entity recognition [56] related to a location. We use this mechanism to enrich the Twitter data with pictures and videos gained from Flickr and YouTube as those items are very sparely annotated with geo-tags but valuable for emergency management [57]. This allows us to combine input from different social media platforms, e.g., pure textual information but also textual annotation of visual items. Our system has the possibility to include live data from the incident site (e.g., collected directly from first responders). In the

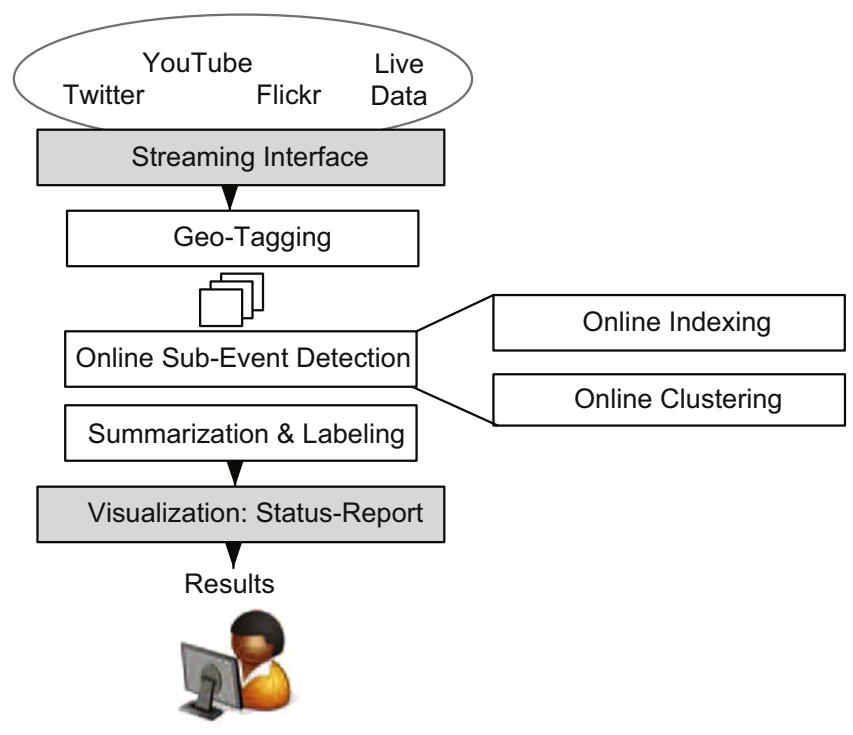

Fig. 1. Multimedia exploration framework. future, we plan to involve additional sources, e.g., data gained from news repositories.

Sub-event detection is based on online feature selection and online clustering as described in the subsequent sections. Section 5 shows an overview of the combination of online indexing and online clustering. The identification of sub-events is based on predefined time periods. The identified sub-events are labeled using the most-relevant terms in the corresponding cluster centers (summarization and labeling stage) and the results are stored in a database for later usage, e.g., browsing.

The sub-events are plotted into a map and visualized to the user in a web-based interface (see Fig. 6). Via a timeline it is possible to browse also historic periods. By clicking on a sub-event, additional information appears in the interface (i.e., all tweets, pictures and videos related to this sub-event). A filtering mechanism allows highlighting of sub-events containing specific keywords.

\section{Online sub-event detection}

For our detection process we identified two (online) processing steps: (i) real-time term addition and removal, (ii) online clustering to identify sub-events from streaming data. As a result of the dynamic evolution of an emergency situation, sub-events may evolve and vanish, and new sub-events may emerge over time. This evolution is dictated by the incoming data items collected from the social media platforms. That is, the vocabulary (i.e., used terms) for describing the incidents changes over time. Therefore, a dynamic indexing approach is needed to continuously track the evolution of terms.

We implemented three indexing approaches, including our own "Learn \& Forget Model" indexing approach. For online processing, the following general steps performed after each batch of items can be identified (extended from [5]):

- Geo-tagging (see Section 6): Items from Flickr and YouTube are automatically tagged in order to identify the geo-coordinates (tweets are already tagged).

- Online indexing (for details see Section 7):

- Standard incremental term frequency-inverse document frequency (tf-idf) as used in Brants et al. [46] and described in Section 7.1.

- Skewness for detecting "bursty" keywords which are then used as terms/features for clustering. Skewness is one of the criteria used by Lee et al. [48] for bursty keywords detection (details in Section 7.2).

- Our "Learn \& Forget Model" ([5], Section 7.3). The terms own weights. These weights are used to identify important and non-important terms.

- Index augmentation:

- By using the different selection mechanisms, incoming data items (i.e., retained terms from the previous step) are adopted into vector representations.

- Geo-data are added to the term vector representations.

- Online clustering (see Section 8):

- Existing cluster representations (from the previous step) are adapted based on the new term set (i.e., outdated ones are removed and new important terms are added).

- The new data items of the batch are clustered.

- Visualization (see Fig. 6, Section 9):

- Sub-events/clusters are labeled with the most important terms and visualized in a map of the web-based userinterface.

- Browsing functionalities are offered to explore the results and filter additional information. 
The next sections describe the geo-tagging and the indexing methods currently implemented in our framework. Additionally, the online clustering algorithm and its adaptation to the changing terms is described as well.

\section{Geo-tagging}

To identify the location of picture and video items, we use geotagging to tag new items by means of named entity recognition [56]. The location entities come with the textual metadata (i.e., title, description, and tags) of Flickr and YouTube. The first two locations (denoted as $L_{1}$ and $L_{2}$, e.g., Manhattan, NY) of an item are used to assign coordinates. If no location information is found in the textual metadata, the user location - if available - is used. If no location is found at all, the item is discarded from further processing.

We search first for the location in the text instead of taking the user location into account, because people can write about things independent of their home location, e.g., focusing on their current location.

Coordinates (related to the identified locations $L_{1}$ and $L_{2}$ ) are searched by accessing the geographical database Geo-Names.org ${ }^{1}$. The provided GeoNames WebService offers the possibility to perform either a search within the database or through Wikipedia to extract/obtain coordinates.

To support geo-tagging, we also include a context $\kappa$ into the search string (e.g., USA) to limit the problem of identifying cities with the same name in other countries outside the given context. In general, the context (e.g., the affected country or city) is known in an emergency situation. This could be changed on the fly if the context changes, i.e., the crisis shifts to another country or area.

The geo-tagging tries to determine the coordinates by performing the following processing sequence: (1) combine all identified locations plus the context to search for coordinates (i.e., $\left\langle L_{1}, L_{2}, \kappa\right\rangle$ ), (2) search for the first location string combined with the context $\left\langle L_{1}, \kappa\right\rangle$, (3) search for the second location string combined with the context $\left\langle L_{2}, \kappa\right\rangle$.

If it is not possible to identify the coordinates with the given option, in each step, an additional Wikipedia search with this option is performed. The coordinates can be extracted from the related Wikipedia entry. The corresponding item is annotated with the identified coordinates. If no coordinates are determined, the item is discarded from further processing.

\section{Online indexing}

There are already some approaches that take the evolution of terms over time into account. We do not distinguish between different data channels (e.g., between Twitter and Flickr) in calculating the indexing. The reason for that is that we want to identify sub-events based on the current incoming items, independently of any synchronization between those channels. We implemented the following indexing methods.

\subsection{Incremental tf-idf}

In general, one data item is represented as a vector of indexing terms reflecting on the importance of each term for that specific item. The traditional term frequency-inverse document frequency (tf-idf) [58] is used as follows:

$i d f_{t}=\log \frac{N}{d f_{t}}, \quad t f_{-} i d f_{t, d}=t f_{t, d} \cdot i d f_{t}$

\footnotetext{
${ }^{1}$ www.geonames.org [Accessed: September 2014].
}

where $t f_{\_} i d f_{t, d}$ shows the term frequency $t f_{t, d}$ of term $t$ in the document $d$ times the inverse document frequency $i d f_{t}$ of all documents in the corpus containing $t$. The $i d f_{t}$ of term $t$ is calculated based on $N$ representing all known items and $d f_{t}$ that represents the frequency of term $t$ in the $N$ documents.

In an incremental model the document frequencies are adapted or calculated based on new incoming terms. This could be done in two ways [46]. First, a training set is used to identify initial tf-idf values, which change as new documents arrive. Second, it is possible to compute tf-idf values from scratch using incoming documents. In our case, the creation of initial tf-idf values is often not feasible due to the unique characteristics (e.g., different impacts of a crisis due to the nature of the crisis, the affected location, and infrastructure) of an emergency situation and the missing training data. Hence, we implemented the second option where frequencies are calculated from scratch. This means $N$ represents all items seen so far from the system and it continuously updates the $d f$ when new items arrive (i.e., incremental tfidf) as suggested by Brants et al. [46].

Additionally, we remove terms having a very low document frequency (i.e., $\left.d f_{t}<\theta_{d f}\right)^{2}$ as they are known for not being very informative [46]. From the remaining terms, those with the highest tf-idf values are selected. All items from the current batch (period) are represented with the terms extracted from this step.

\subsection{Skewness}

"Bursty words" may help reveal indexing terms from incoming documents to identify sub-events. Lee et al. [48] describe a combined measure, including skewness, to identify such frequent terms in a period of one day. We do not implement all facets of the combined measure, e.g., as it also punishes periodic terms which, in our case, are not seen as irrelevant (e.g., reoccurring situations can have repeating terms, like fire and damage) and therefore should not be punished.

Skewness is a statistical measure which we use in this work to find suitable indexing terms for a period of time; it is calculated as follows [48]:

skewness $_{t, p}=\frac{E\left(x_{t, p}-\mu\left(x_{t, p}\right)\right)^{3}}{\sigma\left(x_{t, p}\right)}$

The skewness of term $t$ is calculated based on a vector $x_{t, p}$ that contains all term frequencies of term $t$ in all known documents $\left(d_{1}, d_{2}, d_{3} \ldots, d_{k}\right)$ in the current period $p$ (i.e., $x_{t, p}=<t f_{t, p, d_{1}}, t f_{t, p}$, $\left.d_{2} \ldots, t f_{t, p, d_{k}}>\right) . \mu$ and $\sigma$ describe the mean and the standard deviation of $x_{t, p}$. The more skewed the distribution function, the higher the values of skweness $s_{t, p}$ [48].

Here, we also remove terms that have a low document frequency (i.e., $d f_{t}<\theta_{d f}$ ). Afterwards, we select terms with the highest skewness for clustering. This gives us the most important indexing terms for the current period. For clustering, we transform the identified indexing term set to the vector space model representation. Hence, we calculate the tf-idf values for each period considering the selected terms.

\subsection{Learn \& Forget $(L \& F)$ term selection}

This method implements a weighted version of the tf-idf. It uses a weighting mechanism for smooth removal of outdated terms and the inclusion of new ones. The tf-idf is calculated for a batch of documents (i.e., calculating $N$ and $d f_{t}$ ) based on a period $p$ (given by the user). The period depends on the nature of the crisis

\footnotetext{
${ }^{2}$ Brants et al. [46] suggest a threshold of $\theta_{d f}=2$.
} 
(e.g., slow or fast moving) and on the characteristics of the data stream (e.g., number of incoming items per time unit).

The additional weighting mechanism for calculating the relevance of each term is based on the incoming documents containing that specific term. We apply a first-order discrete time low pass filter [59, Eq. 8.62] in order to smooth incoming signals. The weights for known terms are refreshed based on Eq. (3a) and (3b). after each sampling interval $k_{s}$ at time $k$. The sampling interval $k_{s}$ has to be defined as a fraction of $p$, e.g., $k_{s}=5 \mathrm{~min}$ and $p=30 \mathrm{~min}$.

$g_{t, k}=(1-\gamma) \cdot u_{t, k}+\gamma \cdot g_{t, k-1}, \quad u_{t, k}>g_{t, k-1}$

$g_{t, k}=(1-\delta) \cdot u_{t, k}+\delta \cdot g_{t, k-1} \quad$ otherwise

$g_{t, k}$ indicates the weight of term $t$ at time $k . g_{t, k-1}$ denotes the weight of the term $t$ from the previous sampling interval at time $k-1$. $u_{t, k}$ is the number of incoming documents containing the term $t$ at sampling time $k$. The first line (Eq. (3a)) of the equation serves to point out the novelty brought by the new items (the smaller $\gamma$, the faster the learning of incoming information). The second line (Eq. (3b)) is used to define the speed of forgetting the indexing terms (the higher $\delta$, the slower the forgetting).

$\gamma$ and $\delta$ are empirical values defined by the emergency manager based on his/her experiences (e.g., fast or slow evolving emergency). We suggest a ratio $\gamma<\delta$ which indicates that a high number of incoming items with term $t$ are learned faster than this term is being forgotten. Fig. 2 shows for different $\gamma, \delta$-settings the behavior of the weighting $g_{t, k}$ for incoming tweets $u_{t, k}$ at each timestamp $t$.

The resulting weights $g_{t, k}$ denote the importance of a term over time and act as memory to remember terms. The importance (Eq. (4)) is given by the ratio between the current valid weight and the maximum weight of the term $t$ reached during the selection phase.

importance $_{t, k}=g_{t, k} / g_{-} \max _{t}$

Terms with the highest value based on Eq. (4) are identified as important and used for clustering in period $p$. Terms that have weights below a certain importance factor are removed from the possible term set. We empirically set this threshold to $\beta=0.2$ meaning that $80 \%$ of the importance have been lost.

The weight is then included in the tf-idf formula (see Eq. (5)) to ensure the smooth removal of terms.

scaled_tf_idf $f_{t, d}=$ importance $_{t, p} \cdot t f_{t, d} \cdot i d f_{t}$

The scaled_tf_idf $f_{t, d}$ for item $d$ and term $t$ considers the importance of term $t$ (see Eq. (4)) at the end of each period $p$. The calculation of

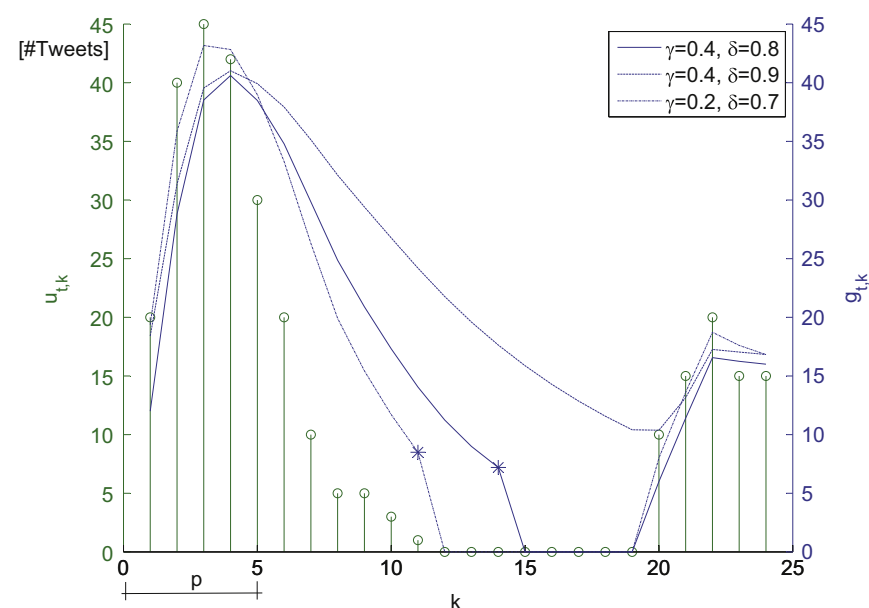

Fig. 2. $g_{t, k}$ vs. $u_{t, k}$ (e.g., incoming tweets) for $p=5 \mathrm{~min}, k_{s}=1 \mathrm{~min}$ with different learning and forgetting factors; removed terms (importance $<0.2$ ) are marked with $\mathrm{a} \star$ the weights is not done after each new item; instead, the incoming items are accumulated considering the sampling time $k$ and clustered after the period $p$.

The changed term set gained from all three indexing methods is also reflected in the clustering. This is done by deleting outdated terms from the clustering prototypes and by adding new ones that are relevant to the new batch.

\section{Online clustering}

Terms identified via the online indexing step are included in the online clustering algorithm after each period. We adapt the Growing Gaussian Mixture Models (2G2M) algorithm [6] to handle complete unlabeled data (see Algorithm 1). Clusters are described as multivariate Gaussians, ${ }^{3}$ where the number of Gaussians changes dynamically taking into account the number of terms in the incoming data. Because sub-event detection is done in an unsupervised way, meaning that social media items are not labeled, the detection algorithm should rely on clustering that does not require any pre-labeled data. Therefore, we adapted the online algorithm described in [6] to our case by removing the steps dealing with the case of labeled data. The following symbols are used in Algorithm 1 [6]:

Algorithm 1. Steps of $2 G 2 M$ handling unlabeled data (adapted from [6])

1: Given a new input $x_{i}$, compute the probability of match of the input with each cluster: $\forall j=1 \ldots K$

$$
\begin{aligned}
& p_{j}= \begin{cases}\tau_{j} \phi_{j}\left(x_{i} ; \mu_{j}, \Sigma_{j}\right) & \text { if } d_{M}\left(x_{i}, \phi_{j}\right)<T_{\Sigma} \\
0 & \text { otherwise }\end{cases} \\
& d_{M}\left(x_{i} ; \phi_{j}\right)=\sqrt{\left(x_{i}-\mu_{j}\right)^{T} \Sigma_{j}^{-1}\left(x_{i}-\mu_{j}\right)}
\end{aligned}
$$

2: Let $R=\left\{j \mid p_{j}>0\right\}$ be the index set of Gaussians matching the input

3: createGaussian $\leftarrow(R==\varnothing)$ (i.e., no match found: $\left.\nexists j, p_{j}>0\right)$

4: if not createGaussian then

5: Compute the index of best matching Gaussian:

$$
w=\underset{j=1 \ldots K}{\arg \max }\left\{p_{j}\right\}
$$

6: $\quad$ Compute the expected posterior:

$$
q_{w}=\frac{p_{w}}{\sum_{k=1 \ldots K} p_{k}}
$$

7: Update the parameters of the Gaussian:

$$
c_{w}=c_{w}+q_{w}
$$

$\tau_{w}(t)=(1-\alpha) \tau_{w}(t-1)+\alpha q_{w}$

$\eta_{w}=q_{w}\left(\frac{1-\alpha}{c_{w}}+\alpha\right)$

$\mu_{w}(t)=\left(1-\eta_{w}\right) \mu_{w}(t-1)+\eta_{w} x_{i}$

$\Sigma_{w}(t)=\left(1-\eta_{w}\right) \Sigma_{w}(t-1)+\eta_{w}\left(x_{i}-\mu_{w}(t-1)\right)^{2}$

8: end if

9: if createGaussian then

10: Decay the weight of all Gaussians

$$
\forall j=1 \ldots K, \tau_{j}(t)=(1-\alpha) \tau_{j}(t-1)
$$

11: $\quad$ Remove the least contributing Gaussian and create a new one initialized with the new input:

$$
\begin{aligned}
& m=\underset{j}{\arg \min }\left\{\tau_{j}\right\} \\
& (\tau, c, \mu, \Sigma)_{m}=\left(\alpha, 1, x_{i}, \Sigma_{0}\right)
\end{aligned}
$$

\footnotetext{
${ }^{3}$ Note that we use the Gaussian distribution because we deal with weighted terms and not their count as in multi-nominal distribution.
} 
12: end if

13: Split largest Gaussian if volume $(\mathrm{V})>T_{\text {split }}$

$$
V(\phi(\mu, \Sigma))=\operatorname{det}(\Sigma)
$$

14: Merge closest Gaussians if KL distance (kld) $<T_{\text {merge }}$

$$
\begin{aligned}
\operatorname{kld}\left(\phi_{1}, \phi_{2}\right)= & \log \left(\frac{\left|\Sigma_{2}\right|}{\left|\Sigma_{1}\right|}+\operatorname{tr}\left(\Sigma_{2}^{-1} \Sigma_{1}\right)+\left(\mu_{2}-\mu_{1}\right)^{T}\right) \\
& \Sigma_{1}^{-1}\left(\mu_{2}-\mu_{1}\right)-F \\
& \operatorname{skld}\left(\phi_{1},{ }_{-} p h i_{2}\right)=\frac{1}{2}\left(\operatorname{kld}\left(\phi_{1}, \phi_{2}\right)+\operatorname{kld}\left(\phi_{2}, \phi_{1}\right)\right)
\end{aligned}
$$

15: Normalize the $\tau_{j}^{\prime}$ s

$K \quad$ max. number of clusters $j=1 \ldots K$ (the number of multivariate Gaussians for each cluster)

$F \quad$ current number of features/terms

$\mu_{j} \quad$ mean values for Gaussian describing the features of cluster $j$ (number of means is based on the number of features)

$\phi_{j} \quad$ density of a multivariate Gaussian describing the cluster

$\Sigma_{0} \quad$ initial covariance matrix of the Gaussians $F \times F$

$\tau_{j} \quad$ weight of the cluster $j$

$\alpha \quad$ learning rate

$T_{\Sigma} \quad$ closeness threshold for matching Gaussians

$c_{j} \quad$ expected posterior (used for the expectation maximization method) of each cluster

$x_{i} \quad i$ th input (as vector space model)

$T_{\text {split }}$ threshold for split

$T_{\text {merge }}$ threshold for merge

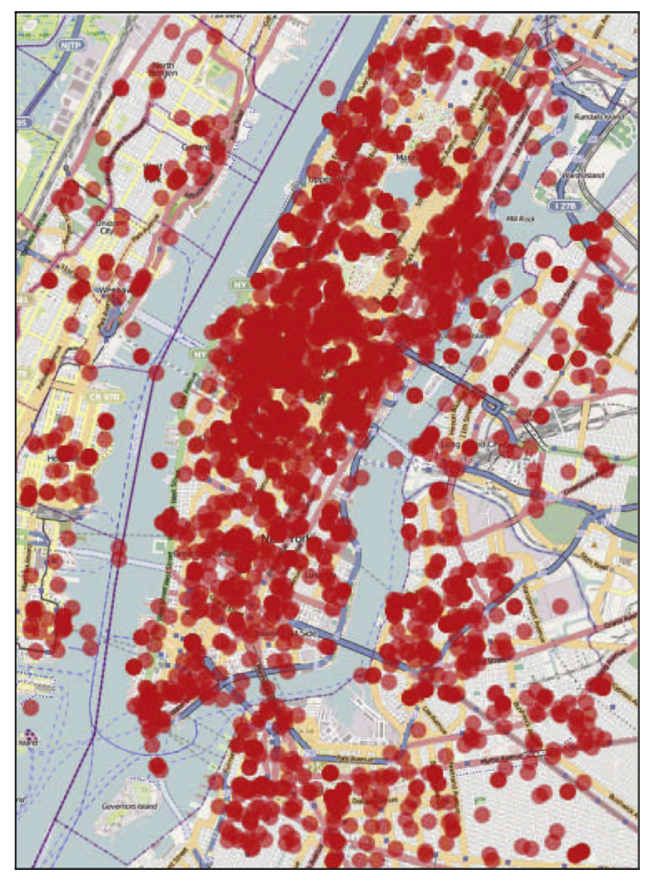

Fig. 3. Distribution of used media items (visualized via OpenStreetMap).
Algorithm 1 starts by comparing the input $x$ with all currently known clusters in the system. A similarity test between the input and the corresponding Gaussians of a cluster is performed using the Mahalanobis distance (Algorithm 1, Step 1: $d_{M}$ in Eq. (7)). If the distance is below the predefined threshold $T_{\Sigma}$, the corresponding cluster is considered as similar to the current input (Algorithm 1, Step 1: $p_{j}$ ).

If many clusters are identified, the index of the most similar Gaussian (i.e., so-called "winner takes all" approach) is retained (Algorithm 1, Step 5). Afterwards, the posterior probability of the winning cluster is calculated and the parameters of this cluster (e.g., weight of the cluster $\phi_{j}$, covariance matrix $\Sigma_{j}$, mean value $\mu_{j}$ ) are adapted based on the input values (Algorithm 1, Steps 6 and 7).

If no matching cluster could be identified, a new Gaussian/ cluster is created using the current input (Algorithm 1, Step 11). Before the new Gaussian is created, the weights of the remaining Gaussians are decayed and the least contributing Gaussian is removed.

The algorithm considers also the merge and split of existing Gaussians to refine the created model (Algorithm 1, Steps 13-15). During clustering, very close clusters are merged. The decision is based on the Kullback-Leibler divergence (see Eq. (19)) [6,60]. If the divergence is below a predefined threshold $T_{\text {merge }}$, they are merged. On the other hand, large clusters are split. A split is performed when the volume in Eq. (18) exceeds a certain threshold $T_{\text {split. }}$. Clusters are split along their dominant principal components [6].

As the indexing terms can change after each batch (period), the clusters have to be adapted. The adaptation to the new term set is performed by updating the Gaussian parameters $\mu_{j}$ and $\Sigma_{j}$ of cluster $j$. When removing terms, the corresponding values (rows/ columns) of these old terms are removed. If there are new terms to consider, zero values for $\mu_{j}$ and $\Sigma_{j}$ are included for each cluster $j$.

\section{Experiments}

For the sake of evaluation, we use real-world data (i.e., Tweets,

\begin{tabular}{|c|c|c|c|c|c|}
\hline \multirow[t]{2}{*}{ Method } & \multirow{2}{*}{$\begin{array}{l}\text { Setting } \\
(\gamma, \delta)\end{array}$} & \multicolumn{2}{|l|}{ DB } & \multicolumn{2}{|l|}{ S } \\
\hline & & 60 & 110 & 60 & 110 \\
\hline \multirow[t]{3}{*}{$\mathrm{L} \& \mathrm{~F}$} & $(0.2,0.6)$ & 6.532 & 9.087 & 0.345 & 0.422 \\
\hline & $(0.5,0.6)$ & 6.524 & 8.033 & 0.359 & 0.426 \\
\hline & $(0.2,0.4)$ & 8.876 & 7.771 & 0.334 & 0.443 \\
\hline Skewness & - & 5.480 & 8.067 & 0.401 & 0.412 \\
\hline inc. tf-idf & - & 5.805 & 8.327 & 0.369 & 0.428 \\
\hline
\end{tabular}
Flickr and YouTube items) from the Hurricane Sandy 2012. In

Table 2

Overview of averaged DB and Silhouette values (60 and 110 terms).

Table 1

Topics of Hurricane Sandy 2012 given in Wikipedia [61].

\begin{tabular}{ll}
\hline Topics & Description (period: 29th October, local time) \\
\hline Airports/flights & Closed airports and canceled flights, i.e., JFK, Newark (8 PM) \\
Evacuation & Several hospitals and FDNY Emergency Medical Services were evacuated \\
Flooding & Different districts, in addition also tunnels (7 PM) and sub-ways \\
Power/electricity & Power outages in several districts, e.g., Manhattan, Queens, Staten Island, Brooklyn, Bronx, etc. \\
Fire & Several fires due to fallen trees or blown-up transformers, e.g., Breezy Point, Queens (approx. 11 PM) \\
Wind & Fallen trees and broken branches, damage due to the heavy wind, crane collapsed, etc. \\
\hline
\end{tabular}


a

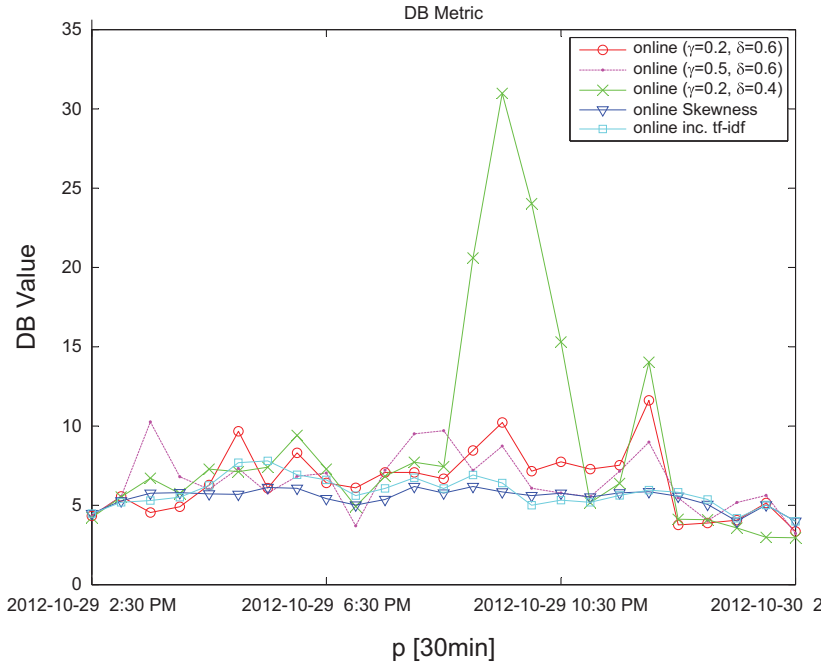

b

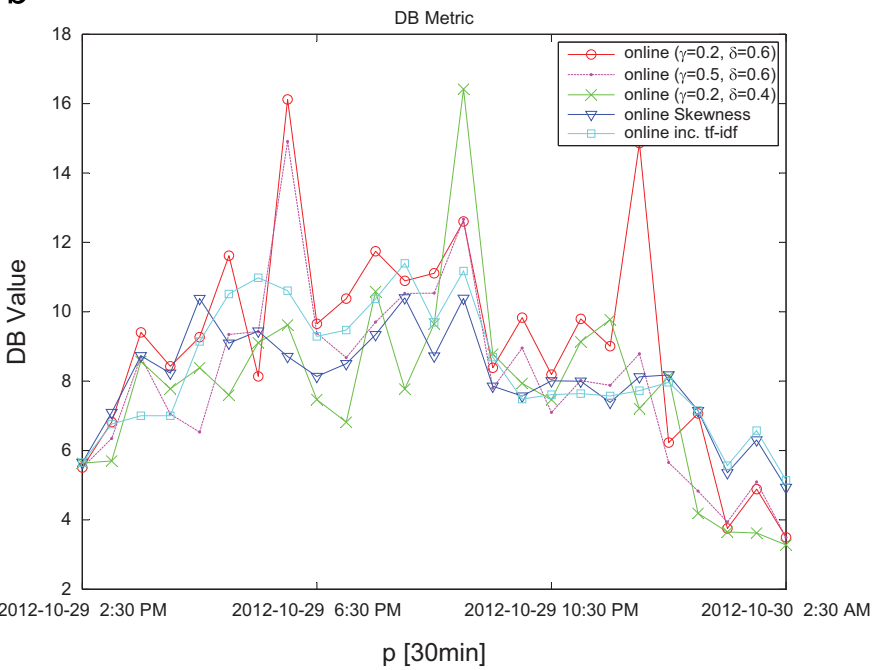

Fig. 4. DB Values (using the local time). (a) DB (60 terms) and (b) DB (110 terms).

a

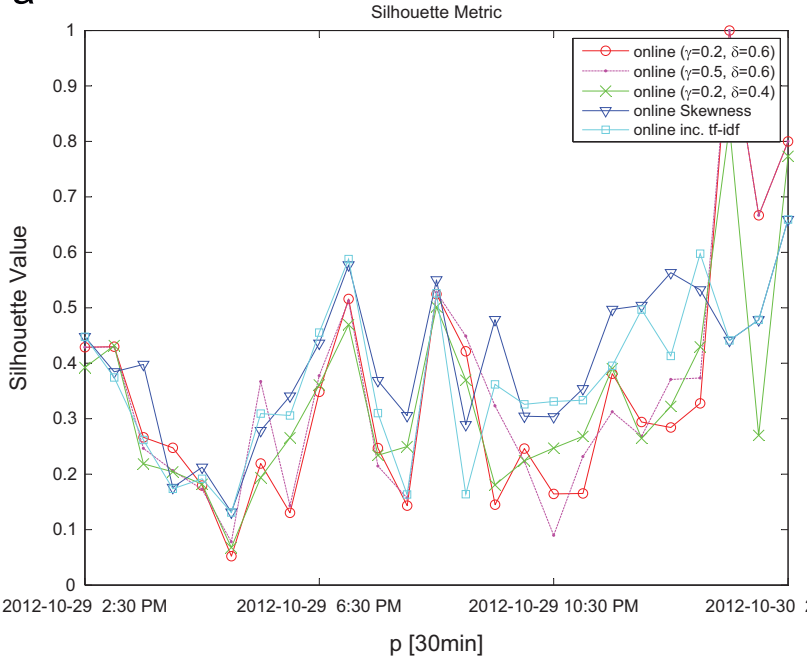

b

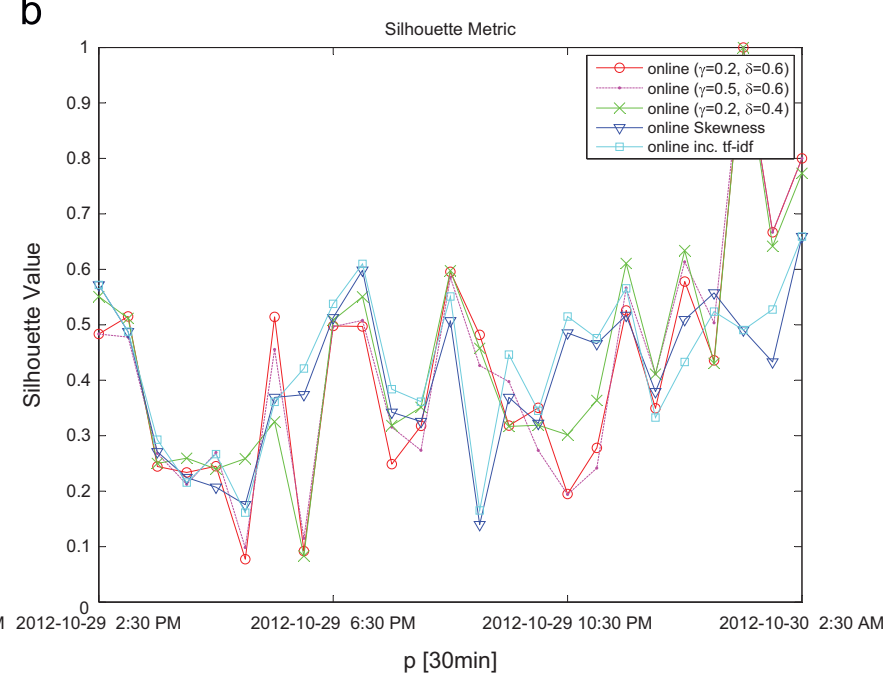

Fig. 5. $S$ Values (using the local time). (a) S (60 terms) and (b) S (110 terms).

particular, we collected items during the main impact of the hurricane from October 29, 2012, till November 1, 2012.

Considering the enormous number of tweets, approximately 3.7 million tweets, we conducted our experiments based on a smaller snapshot to evaluate the approach for its effectiveness. Therefore, we considered only geo-tagged tweets related to the locations around Manhattan, Brooklyn and New Jersey. This results in 1003 tweets for the period from October 29, 02:00 pm to October 30, 02:30 am. Beside the tweets, we also used 286 pictures from Flickr and 167 videos from YouTube. In total, we have a data set consisting of 1456 social media items distributed on the three locations as shown in Fig. 3.

In Table 1, the major topics related to the Hurricane Sandy 2012 are extracted from the report given in Wikipedia [61]. Topics are therefore readily available and are considered to be the reference topics/sub-events. They will be compared against those detected by our sub-event detection approach. The major incidents during the hurricane are related to damages, flooding and power outages.
They can also be found in the results obtained via the tested algorithms (see Section 9.2, Tables 3-5).

\subsection{Description}

We evaluated different online/indexing methods as described in Section 7. In particular, we compare our approach, the Learn \& Forget method (Section 7.3, Pohl et al. [5]), against the incremental tf-idf (Section 7.1, Brants et al. [46]) and the skewness-based method (Section 7.2, Lee et al. [48]). Moreover, different settings (time and parameter) of the Learn \& Forget method are tested.

We consider only nouns (e.g., flood and damage) as possible item candidates. The extracted nouns are stemmed using the common Porter Stemmer [62]. Similar nouns are grouped together and treated as one (e.g., US and USA, flood and flooding). This is performed by making use of the relations between concepts managed in WordNet [63]. The resulting terms are then weighted. 
Table 3

Resulting topics based on the cluster IDs; includes the stem forms of words and markers for important words describing sub-events.

\begin{tabular}{|c|c|}
\hline ID & Labels \\
\hline \multicolumn{2}{|c|}{$\mathrm{L} \& \mathrm{~F}[(\gamma=0.5, \delta=0.6) ; 60$ terms $]$} \\
\hline 1 & new park apocalyps con sandi hurrican \\
\hline 2 & new ny sandi frankenstorm hurrican york power \\
\hline 3 & ny mondai new frankenstorm sandi con hurrican \\
\hline 4 & $\begin{array}{l}\text { hurrican apocalyps new frankenstorm octob sandi } \\
\text { citi }\end{array}$ \\
\hline 5 & hurrican new citi sandi ny other nyc \\
\hline 6 & york nyc park build manhattan sandi fire \\
\hline 7 & new nyc apocalyps explos sandi power station \\
\hline 8 & hurrican other power explos ed part station \\
\hline 9 & abc center evacu am ambul chri edt \\
\hline 10 & wind octob new sandi hurrican york other \\
\hline 11 & sandi citi apocalyps power york con hurrican \\
\hline \multicolumn{2}{|c|}{ Brants [60 terms] } \\
\hline 1 & hurrican sandi dai tunnel power fall manhattan \\
\hline 2 & apocalyps frankenstorm sandi other hurrican \\
\hline 2 & power \\
\hline 3 & newyork nyc park ny sandi storm hurrican \\
\hline 4 & wind crane us storm time build central \\
\hline 5 & street hurrican dai east bridg video power \\
\hline 6 & mondai citi sandi hurrican york new power \\
\hline 7 & nyc citi park mondai evacu new manhattan \\
\hline 8 & sandi hurrican citi york new home jersei \\
\hline 9 & nyc east hurrican \\
\hline 10 & hudson water flood river east coast hit \\
\hline 11 & nyc wind video sandi manhattan us hurrican \\
\hline 12 & rain hurrican sandi ny new us york \\
\hline 13 & $\begin{array}{l}\text { other apocalyps frankenstorm ny sandi york } \\
\text { hurrican }\end{array}$ \\
\hline \multicolumn{2}{|c|}{ Lee [60 terms] } \\
\hline 1 & hurrican sandi dai peopl wai time park \\
\hline 2 & newyork nyc park ny sandi storm hurrican \\
\hline 3 & crane new us storm park time central \\
\hline 4 & nyc sandi hurrican wai york new street \\
\hline 5 & street hurrican dai east manhattan power wai \\
\hline 6 & citi hurrican \\
\hline 7 & nyc oct citi park mondai wai ey \\
\hline 8 & oct mondai sandi hurrican york new power \\
\hline 9 & other apocalyps frankenstorm ny sandi york new \\
\hline 10 & hurrican dai peopl sandi new ny york \\
\hline 11 & $\begin{array}{l}\text { apocalyps frankenstorm sandi other hurrican } \\
\text { power }\end{array}$ \\
\hline 12 & nyc east hurrican \\
\hline 13 & nyc citi jersei flood east coast manhattan \\
\hline 14 & frankenstorm apocalyps other sandi ny york new \\
\hline 15 & nyc wind video sandi manhattan us hurrican \\
\hline
\end{tabular}

We evaluate the clustering using the Silhouette (S) [64] and the Davies-Bouldin (DB) [65] metrics. The S metric identifies how close related items in a specific cluster are. High values of the Silhouette metric indicate good and well-separated clustering.

The Silhouette is expressed as follows for an item $m$ and a cluster $i$ [64]:

$b_{m}=\min _{j \neq i} d_{m, j}$

$s_{m}=\frac{b_{m}-a_{m}}{\max \left\{a_{m}, b_{m}\right\}}$

$a_{m}$ represents the average dissimilarity of the item $m$ to all other items in the same cluster $i . d_{m, j}$ describes the average dissimilarity of all items from the other clusters $j$ to $m$. $b_{m}$ represents the smallest dissimilarity of $m$ to $d_{m, j}$. The $S$ value of a cluster is the average of $s_{m}$ from each item $m$ in the cluster.

The DB index is expressed as follows [65]:

$D B=\frac{1}{n} \sum_{i=1}^{n} R_{i}$ where $R_{i}=\max _{j=1, \ldots, n ; j \neq i} R_{i j}, \quad i=1, \ldots, n$

$R_{i j}=\frac{s_{i}+s_{j}}{d_{i j}}$

The DB index describes the similarity between clusters based on the dispersion $s_{i}$ of a cluster $c_{i}$ and by considering $d$ as dissimilarity measure between two clusters [65]. Small values of DB indicate a good clustering emphasizing that clusters are dissimilar to each other.

The parameters for the clustering algorithm (shown in Algorithm 1) are empirically evaluated and set for all three indexing methods

to $\alpha=0.01, K=1000, \tau_{\sigma}=3, T_{\text {merge }}=8, T_{\text {split }}=20$.

\subsection{Comparison of the results}

In all experiments, the number of terms retained is the same for all methods. Two experiments ( 60 terms, 110 terms) have been performed. For the Learn \& Forget (L\&F) method, several settings have been tested. Table 2 shows the average DB and $S$ values over all periods $(p=30 \mathrm{~min}$ ).

In addition, Figs. 4 and 5 outline the behavior of the different methods when data comes over time for different settings. The

Table 4

Resulting topics based on the cluster IDs; includes the stem forms of words and markers for important words describing sub-events.

\begin{tabular}{ll}
\hline ID & Labels \\
\hline L\&F $[(\gamma=0.2, \delta=0.4) ; 60$ terms $]$ \\
1 & wind york park build part sandi fire \\
2 & hurrican apocalyps new frankenstorm sandi york \\
& manhattan \\
3 & new nyc part explos sandi manhattan station \\
4 & new hurrican sandi nyc park citi \\
5 & hurrican manhattan power explos ed station \\
6 & evacu \\
7 & octob con new sandi hurrican york power \\
8 & mondai abc center am ambul chri edt \\
& sandi nyc citi manhattan york hurrican new \\
\hline
\end{tabular}

Table 5

Resulting topics based on the cluster IDs; includes the stem forms of words and markers for important words describing sub-events.

\begin{tabular}{ll}
\hline ID & Labels \\
\hline L\&F $[(\gamma=0.2, \delta=0.4) ; 110$ terms $]$ & park ny other brooklyn coast sandi evacu \\
1 & citi explos other power ny sandi york \\
2 & york park build video con part evacu \\
3 & other coast video frankenstorm part sandi storm \\
4 & hurrican apocalyps other sandi east york \\
5 & manhattan \\
& new storm hurrican frankenstorm apocalyps ed \\
6 & station \\
7 & nyc other bitch alealeeeoop boss apocalyps \\
8 & manhattan abc center am ambul chri edt \\
9 & citi other mondai explos power sandi apocalyps \\
10 & manhattan abc center am ambul chri edt \\
11 & other citi apocalyps power sandi video coast \\
12 & hurrican apocalyps other sandi video mondai \\
& power \\
13 & street octob bit asi condado other sandi \\
14 & other coast 72nd halloween st frankestorm \\
15 & instacollag \\
16 & wind nyc storm water flood lower attack \\
17 & manhattan abc center am ambul chri \\
& high other lic dry gotta laugh apocalyps \\
\end{tabular}




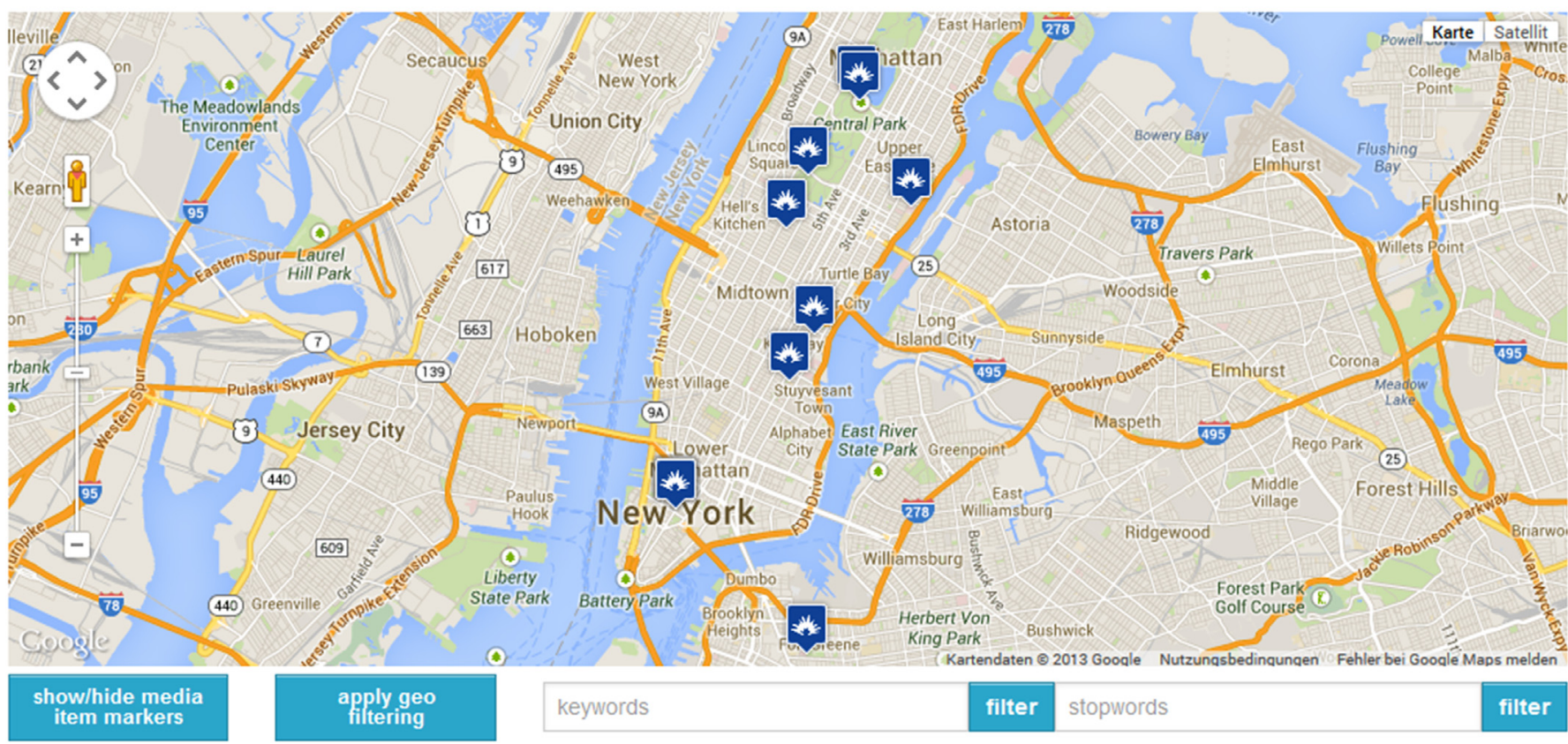

\section{timeline}

\section{《 30.10.2012: 03:30 30.10.2012:04:00 30.10.2012: 04:30 30.10.2012:05:00 30.10.2012:05:30 $30.10 .2012: 06: 00 \quad 3 \mathrm{C}$}

Fig. 6. The application shows sub-events for L\&F $[(\gamma=0.5, \delta=0.6) ; 60$ terms] in UTC time 05:30 pm (Note: sub-events can overlap on the map). Markers by MapIconCollection mapicons.nicolasmollet.com.

methods exhibit little variation when using different clustering evaluation measures, in the case of DB and S. Therefore, changing the measure does not imply any clear response trend of the algorithms.

Table 2 summarizes the metrics for different term settings (60 and 110) indicating the best results for each term setting in bold. By increasing the number of terms from 60 to 110 , the results for the L\&F improve (see Table 2, column 110 terms). In general, all three methods show very similar outcomes (see also the DB and S values in Figs. 4 and 5), but differ in the way features are selected (see Table 3 [60 terms]).

Tables 3-5 show the results of the different approaches and settings. Terms are given in their stem form that the natural language processor (NLP) outputs. To support their interpretation, the stems can be mapped onto the topics' description in Table 1. Bold terms indicate important terms identified by one approach only. Italic terms indicate terms identified by all approaches. In the following sections, the most important settings are compared with each other and the differences are highlighted.

Given Table 3, the L\&F method identifies additional terms and hence uncovers specialized sub-events (topics) compared to the other approaches. For example, for the period 00:30 local time (05:30 UTC) on October 30 (this period shows also small DB values in Fig. 4(a)), information regarding fire, evacuation and explosion was extracted (see Table 3 ). The "explosion" was only labeled by the L\&F method, also information regarding fire services was only extracted by our approach. The incremental tf-idf approach identified a topic concerning the evacuation. Skewness identifies none of these concepts, but together with the inc. tf-idf an additional item documenting the flooding in Lower Manhattan is detected. Information regarding power outages or comments on power supply are covered by all approaches ("power" in Table 3). Additionally, the L\&F method achieves a smaller number of subevents. A visualization of the sub-events identified by L\&F can be found in Fig. 6. It also identifies topics regarding canceled flights in another period at 02:00 UTC (not shown in this table).

The L\&F for the $(\gamma=0.2, \delta=0.4)$ setting shows that the terms are learned and forgotten very fast (see Table 4). This results in a peak in the DB diagram, as the terms in the term set are changing fast too (especially for specific periods). The L\&F with $\gamma=0.2$ and $\delta=0.4$ also identifies important terms, but aggregates items too strong/tight.

When increasing the number of terms as the Information Retrieval (IR) literature suggests, we noticed that the L\&F method increases its performance. Results for the increased number of terms (110 terms) can also be found in Table 2. Details on the values of the metrics for each period can be found in Figs. 4 and 5 (on the right-hand side). It can be seen that for a high number of terms the L\&F approach improves its performance. The L\&F method $(\gamma=0.2, \delta=0.4$ ) shows the best performance as more descriptive terms are included. More clusters are generated as additional information becomes available. For the period 05:30 a topic regarding the sub-event "flood" is identified (see Cluster 15 in Table 5). In summary, 17 sub-events are uncovered (as shown Table 5) compared to the 8 sub-events extracted with 60 terms in Table 4.

Table 2 shows that for an increased number of terms (from 60 to 110 terms) the $\mathrm{L} \& \mathrm{~F}(\gamma=0.2, \delta=0.4)$ approach improves performance compared to the other approaches. The DB value declines whereas the $\mathrm{S}$ value increases. Based on the $\mathrm{DB}$ and $\mathrm{S}$ results given in Tables 2 and 5 the setting $[(\gamma=0.2, \delta=0.4) ; 110$ terms] provides the best results in this study. 


\subsection{Discussion and future work}

The online indexing methods show similar behavior as can be seen in Figs. 4 and 5. They identify in general important sub-events related to flights, damage, flood, evacuation, power and different relief units. These major topics can also be found in Table 1. Increasing the number of terms shows also an increased performance for the L\&F method (see Table 2). This method has advantages as it does not need to store all terms found in the data stream (see incremental tf-idf). Irrelevant terms are removed from the index set. In addition, the L\&F method allows us to control learning and forgetting of the terms by means of the parameters. This makes the method flexible for adapting to new situations (e.g., fast or slow changes) that can emerge during a crisis. Additionally, it allows us to memorize terms over periods. In contrast, the skewness method cannot identify easily terms over different periods.

In the future, we aim to devise an automatic method to adjust parameters for the clustering algorithm (i.e., recognizing conceptual drifts in the incoming information, the nature of the stream, etc.). It is also possible to incorporate visual features, especially from pictures, and extend the algorithm to handle those features appropriately.

\section{Conclusion}

This paper presents a framework for identifying sub-events based on crisis-related data. The identification uses a dynamic indexing and an online clustering algorithm. We investigated three online indexing methods (incremental tf-idf, skewness and Learn \& Forget). The experiments show that for a higher number of indexing terms the Learn \& Forget method performs better than the incremental tf-idf and skewness methods. Sub-events related to important incidents can be identified, like power outage, flood, evacuation, etc. A demonstration performed in September 2013 also showed the usefulness of the suggested framework during a real-time emergency response exercise. In the future, we will focus on an automatic method to identify and handle concept drifts in the data, e.g., by re-initializing the algorithm or by adjusting the Learn \& Forget parameters.

\section{Acknowledgements}

The research leading to these results has received funding from the European Union Seventh Framework Programme (FP7/20072013) under Grant agreement no. 261817 and was partly performed in the Lakeside Labs research cluster at Alpen-AdriaUniversität Klagenfurt.

\section{References}

[1] L. Palen, Online social media in crisis events, EDUCAUSE Q. (EQ) 31 (3) (2008) 76-78 $\langle$ http://www.educause.edu/ $\rangle$.

[2] S. Vieweg, A.L. Hughes, K. Starbird, L. Palen, Microblogging during two natural hazards events: what twitter may contribute to situational awareness, in: Proceedings of the 28th International Conference on Human Factors in Computing Systems, CHI '10, ACM, New York, NY, USA, 2010, pp. 1079-1088.

[3] R. Westbrook, T. Karlgaard, C. White, J. Knapic, A holistic approach to evaluating social media's successful implementation into emergency management operations: applied research in an action research study, Int. J. Inf. Syst Crisis Response Manag. 4 (2012) 1-13.

[4] D. Pohl, A. Bouchachia, H. Hellwagner, Automatic sub-event detection in emergency management using social media, in: First International Workshop on Social Web for Disaster Management (SWDM), in conjunction with WWW'12, ACM, Lyon, France, 2012, pp. 683-686.

[5] D. Pohl, A. Bouchachia, H. Hellwagner, Online processing of social media data for emergency management, in: International Conference on Machine Learning and Applications (ICMLA), vol. 2, 2013, pp. 333-338. doi:http://dx.doi.org/ 10.1109/ICMLA.2012.170.
[6] A. Bouchachia, C. Vanaret, GT2FC: an online growing interval type-2 selflearning fuzzy classifier, IEEE Trans. Fuzzy Syst. 22 (4) (2014) 999-1018.

[7] M. Gao, V.K. Singh, R. Jain, Eventshop: from heterogeneous web streams to personalized situation detection and control, in: Proceedings of the 3rd Annual ACM Web Science Conference, WebSci '12, ACM, New York, NY, USA, 2012, pp. 105-108.

[8] V. Lampos, N. Cristianini, Nowcasting events from the social web with statistical learning, ACM Trans. Intell. Syst. Technol. 3 (4) (2012) 72:1-72:22.

[9] V. Lampos, N. Cristianini, Tracking the flu pandemic by monitoring the social web, in: International Workshop on Cognitive Information Processing (CIP), 2010, pp. 411-416. doi:http://dx.doi.org/10.1109/CIP.2010.5604088.

[10] M. Krstajic, C. Rohrdantz, M. Hund, A. Weiler, Getting there first: real-time detection of real-world incidents on twitter, in: 2nd Workshop on Interactive Visual Text Analytics: Task-Driven Analysis of Social Media Content with Visweek'12, 2012, pp. 1-4.

[11] D. Chakrabarti, K. Punera, Event summarization using tweets, in: Proceedings of the International AAAI Conference on Weblogs and Social Media, 2011, pp. 66-73.

[12] C. Shen, F. Liu, F. Weng, T. Li, A participant-based approach for event summarization using twitter streams, in: Proceedings of NAACL-HLT, 2013, pp. 1152-1162.

[13] A. Marcus, M.S. Bernstein, O. Badar, D.R. Karger, S. Madden, R.C. Miller, Twitinfo: aggregating and visualizing microblogs for event exploration, in: Proceedings of the 2011 Conference on Human Factors in Computing Systems, CHI '11, ACM, New York, NY, USA, 2011, pp. 227-236.

[14] B. Klein, X. Laiseca, D. Casado-Mansilla, D. López-de Ipina, A. Nespral, Detection and extracting of emergency knowledge from twitter streams, in: J. Bravo, D. López-de Ipina, F. Moya (Eds.), Ubiquitous Computing and Ambient Intelligence, Lecture Notes in Computer Science, Springer, Berlin, Heidelberg, 2012, pp. 462-469.

[15] M. Cataldi, L. Di Caro, C. Schifanella, Emerging topic detection on twitter based on temporal and social terms evaluation, in: Proceedings of the 10th International Workshop on Multimedia Data Mining, MDMKDD '10, ACM, New York, NY, USA, 2010, pp. 4:1-4:10.

[16] J. Allan, R. Papka, V. Lavrenko, On-line new event detection and tracking, in: Proceedings of the 21st International ACM SIGIR Conference on Research and Development in Information Retrieval, SIGIR '98, ACM, New York, NY, USA, 1998, pp. 37-45.

[17] R. Nallapati, A. Feng, F. Peng, J. Allan, Event threading within news topics, in: Proceedings of the 13th ACM International Conference on Information and Knowledge Management, CIKM '04, ACM, New York, NY, USA, 2004, pp. 446-453.

[18] M. Osborne, S. Petrović, R. McCreadie, C. Macdonald, O. Iadh, Bieber no more: first story detection using twitter and wikipedia, in: Proceedings of SIGIR 2012 Workshop on Time-aware Information Access, 2012, pp. 1-4.

[19] B. O'Connor, M. Krieger, D. Ahn, Tweetmotif: exploratory search and topic summarization for twitter, in: Proceedings of the Fourth International AAAI Conference on Weblogs and Social Media, 2010, pp. 384-385.

[20] K. Starbird, Digital volunteerism during disaster: crowdsourcing information processing, in: Conference on Human Factors in Computing Systems, 2011, pp. 1-4.

[21] F. Abel, C. Hauff, G.-J. Houben, R. Stronkman, K. Tao, Twitcident: fighting fire with information from social web streams, in: Proceedings of the 21st International Conference Companion on World Wide Web, WWW '12 Companion, ACM, New York, NY, USA, 2012, pp. 305-308.

[22] J. Rogstadius, V. Kostakos, J. Laredo, M. Vukovic, A real-time social media aggregation tool: reflections from five large-scale events, in: ECSCW 2011 CSCWSmart? Collective Intelligence and CSCW in Crisis Situations, 2011, pp. 1-9.

[23] L. Chen, A. Roy, Event detection from Flickr data through wavelet-based spatial analysis, in: Proceedings of the 18th ACM Conference on Information and Knowledge Management, CIKM '09, ACM, New York, NY, USA, 2009, pp. 523-532.

[24] R. Fontugne, K. Cho, Y. Won, K. Fukuda, Disasters seen through Flickr cameras, in: Proceedings of the Special Workshop on Internet and Disasters, SWID '11, ACM, New York, NY, USA, 2011, pp. 5:1-5:10.

[25] T. Rattenbury, N. Good, M. Naaman, Towards automatic extraction of event and place semantics from Flickr tags, in: Proceedings of the 30th Annual International ACM SIGIR Conference on Research and Development in Information Retrieval, SIGIR '07, ACM, New York, NY, USA, 2007, pp. 103-110.

[26] X. Liu, R. Troncy, B. Huet, Using social media to identify events, in: Proceedings of the 3rd ACM SIGMM International Workshop on Social Media, ACM, New York, NY, USA, 2011, pp. 3-8.

[27] G. Petkos, S. Papadopoulos, E. Schinas, Y. Kompatsiaris, Graph-based multimodal clustering for social event detection in large collections of images, in: C. Gurrin, F. Hopfgartner, W. Hurst, H. Johansen, H. Lee, N. O'Connor (Eds.), MultiMedia Modeling, Lecture Notes in Computer Science, vol. 8325, Springer International Publishing, Switzerland, 2014, pp. 146-158.

[28] M. Rabbath, P. Sandhaus, S. Boll, Analysing facebook features to support event detection for photo-based facebook applications, in: Proceedings of the 2nd ACM International Conference on Multimedia Retrieval, ICMR '12, ACM, New York, NY, USA, 2012, pp. 11:1-11:8.

[29] Z.-J. Zha, H. Zhang, M. Wang, H. Luan, T.-S, Chua, Detecting group activities with multi-camera context, IEEE Trans. Circuits Syst. Video Technol. 23 (5) (2013) 856-869.

[30] Y. Ke, R. Sukthankar, M. Hebert, Event detection in crowded videos, in: IEEE 11th International Conference on Computer Vision (ICCV), 2007, pp. 1-8. 
[31] C. Schüldt, I. Laptev, B. Caputo, Recognizing human actions: a local SVM approach, in: Proceedings of the 17th International Conference on Pattern Recognition (ICPR), vol. 3, 2004, pp. 32-36.

[32] J. Aggarwal, M. Ryoo, Human activity analysis: a review, ACM Comput. Surv. (CSUR) 43 (3) (2011) 16-1-16:43.

[33] Z.-J. Zha, L. Yang, T. Mei, M. Wang, Z. Wang, T.-S. Chua, X.-S. Hua, Visual query suggestion: towards capturing user intent in internet image search, Commun. Appl. (TOMM) 6 (3) (2010) 13:1-13:19.

[34] Y. Chen, J.Z. Wang, R. Krovetz, Content-based image retrieval by clustering, in: Proceedings of the 5th ACM SIGMM International Workshop on Multimedia Information Retrieval, MIR '03, ACM, New York, NY, USA, 2003, pp. 193-200.

[35] C. Papagiannopoulou, V. Mezaris, Concept-based image clustering and summarization of event-related image collections, in: Proceedings of the 1st ACM International Workshop on Human Centered Event Understanding from Multimedia, HuEvent'14, ACM, New York, NY, USA, 2014, pp. 23-28.

[36] A. Jaffe, M. Naaman, T. Tassa, M. Davis, Generating summaries and visualization for large collections of geo-referenced photographs, in: Proceedings of the 8th ACM Int'l Workshop on Multimedia Information Retrieval, MIR '06, ACM, New York, NY, USA, 2006, pp. 89-98.

[37] I.H. Witten, E. Frank, M.A. Hall, Data Mining: Practical Machine Learning Tools and Techniques: Practical Machine Learning Tools and Techniques, Elsevier, San Francisco, 2011.

[38] M. Hall, E. Frank, G. Holmes, B. Pfahringer, P. Reutemann, I.H. Witten, The WEKA data mining software: an update, SIGKDD Explor. Newsl. 11 (2009) $10-18$.

[39] Y. Yang, J.O. Pedersen, A comparative study on feature selection in text categorization, in: Proceedings of the International Conference on Machine Learning, ICML '97, Morgan Kaufmann Publishers Inc., San Francisco, CA, USA, 1997, pp. 412-420

[40] T. Liu, S. Liu, Z. Chen, W.-Y. Ma, An evaluation on feature selection for text clustering, in: Proceedings of the 20th International Conference on Machine Learning, Washington DC, 2003, pp. 488-495.

[41] H. Liu, L. Yu, Toward integrating feature selection algorithms for classification and clustering. IEEE Trans. Knowl. Data Eng. 17 (4) (2005) 491-502.

[42] M. Dash, K. Choi, P. Scheuermann, H. Liu, Feature selection for clustering-a filter solution, in: Proceedings of IEEE International Conference on Data Mining, 2002, pp. 115-122.

[43] F. Beil, M. Ester, X. Xu, Frequent term-based text clustering, in: Proceedings of the International Conference on Knowledge Discovery and Data Mining, KDD '02, ACM, New York, NY, USA, 2002, pp, 436-442.

[44] A. Ahmed, E.P. Xing, Timeline: a dynamic hierarchical Dirichlet process model for recovering birth/death and evolution of topics in text stream, in: Proceedings of the Twenty-Sixth Conference on Uncertainty in Artificial Intelligence, 2010, pp. 1-10.

[45] S. Alelyani, J. Tang, H. Liu, Data clustering: algorithms and applications, in: Feature Selection for Clustering: A Review, CRC Press, Boca Raton 2013, pp. $1-33$.

[46] T. Brants, F. Chen, A. Farahat, A system for new event detection, in: Proceedings International ACM SIGIR Conference on Research and Development in Information Retrieval, SIGIR '03, ACM, New York, NY, USA, 2003, pp. 330-337.

[47] S. Khy, Y. Ishikawa, H. Kitagawa, A novelty-based clustering method for on-line documents, World Wide Web 11 (2008) 1-37.

[48] S. Lee, S. Lee, K. Kim, J. Park, Bursty event detection from text streams for disaster management, in: Proceedings of International Conference Companion on World Wide Web, WWW '12 Companion, ACM, New York, NY, USA, 2012, pp. 679-682.

[49] M. Devaney, A. Ram, Efficient feature selection in conceptual clustering, in: Proceedings of the Fourteenth International Conference on Machine Learning, ICML '97, Morgan Kaufmann Publishers Inc., San Francisco, CA, USA, 1997, pp. 92-97.

[50] A. Singhal, M. Mitra, C. Buckley, Learning routing queries in a query zone, in: Proceedings of the 20th Annual International ACM SIGIR Conference on Research and Development in Information Retrieval, SIGIR '97, ACM, New York, NY, USA, 1997, pp. 25-32.

[51] A. Bouchachia, R. Mittermeir, Towards incremental fuzzy classifiers, Soft Comput. 11 (2) (2007) 193-207.

[52] X. Wu, K. Yu, H. Wang, W. Ding, Online streaming feature selection, in: International Conference on Machine Learning, 2010, pp. 1159-1166.

[53] S.-U. Guan, S. Li, Incremental learning with respect to new incoming input attributes, Neural Process. Lett. 14 (3) (2001) 241-260.

[54] T. Rückstieß, C. Osendorfer, P. van der Smagt, Minimizing data consumption with sequential online feature selection, Int. J. Mach. Learn. Cybern. 4(3) (2013) 235-243.

[55] I. Katakis, G. Tsoumakas, I. Vlahavas, On the utility of incremental feature selection for the classification of textual data streams, in: P. Bozanis, E. Houstis (Eds.), Advances in Informatics, Lecture Notes in Computer Science, vol. 3746, Springer, Berlin, Heidelberg, New York, 2005, pp. 338-348.
[56] The Stanford Natural Language Processing Group, Named Entity Recognition (NER) and Information Extraction (IE), February 2013. 〈nlp.stanford.edu/ner/ index.shtml>.

[57] S. Liu, L. Palen, J. Sutton, A. Hughes, S. Vieweg, In search of the bigger picture: the emergent role of on-line photo-sharing in times of disaster, in: Proceedings of the Information Systems for Crisis Response and Management Conference, 2008, pp. 140-149.

[58] C.D. Manning, P. Raghavan, H. Schütze, Introduction to Information Retrieval, Cambridge University Press, New York, 2008.

[59] P.D. Cha, J.I. Molinder, Fundamentals of Signals and Systems: A Building Block Approach, Cambridge University Press, New York, 2006.

[60] D. Schnitzer, A. Flexer, G. Widmer, M. Gasser, Islands of Gaussians: the self organizing map and gaussian music similarity features, in: J.S. Downie, R.C. Veltkamp (Eds.), Proceedings of the 11th International Society for Music Information Retrieval Conference (ISMIR 2010), International Society for Music Information Retrieval, 2010, pp. 327-332.

[61] Wikipedia Article, Effects of Hurricane Sandy in New York, 〈http://en.wikipe dia.org/wiki/Effects_of_Hurricane_Sandy_in_New_York $\rangle$, August 2012

[62] M.F. Porter, An algorithm for suffix stripping, Progr.: Electron. Libr. Inf. 14 (1980) 130-137.

[63] C. Fellbaum, WordNet: An Electronic Lexical Database, MIT Press, Cambridge 1998.

[64] P.J. Rousseeuw, Silhouettes: a graphical aid to the interpretation and validation of cluster analysis, J. Comput. Appl. Math. 20 (1987) 53-65.

[65] S. Theodoridis, K. Koutroumbas, Pattern Recognition, Elsevier/Academic Press, San Diego, 2006.

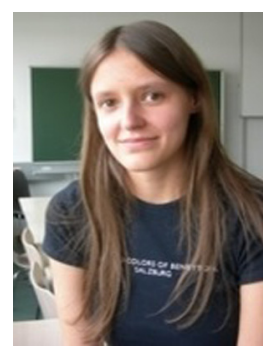

Daniela Pohl received her Dipl.-Ing. (Master's degree) in Computer Science in 2008 at the Alpen-AdriaUniversität Klagenfurt, Austria. She is currently a research assistant and Ph.D. candidate at the Institute of Information Technology, Alpen-Adria-Universität Klagenfurt. She works in the scope of the EU-funded FP7 project BRIDGE (www.bridgeproject.eu) to develop technical solution to improve crisis and emergency management. Her research interests include socia media analysis, information retrieval, data mining, and machine learning.

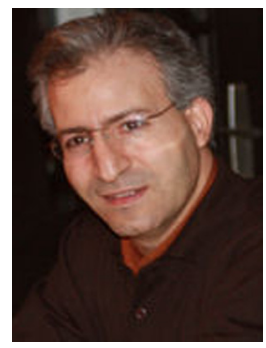

Abdelhamid Bouchachia is currently an Associate Professor at Bournemouth University, Department of Computing, Smart Technology Research Centre, UK. His major research interests include Machine Learning and Soft Computing with a particular focus on online/ incremental learning, semi-supervised learning, prediction systems, and uncertainty modeling. He is the general chair of the International Conference on Adaptive and Intelligent Systems (ICAIS). He serves as a program committee member for many conferences. He also serves as an Associate Editor of Evolving Systems and acts as a member of Evolving Intelligent Systems (EIS) Technical Committee (TC) of the IEEE Systems Man and Cybernetics Society, the IEEE Task-Force for Adaptive and Evolving Fuzzy Systems and the IEEE Computational Intelligence Society.

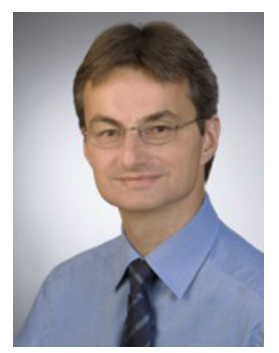

Hermann Hellwagner is a full professor of Informatics in the Institute of Information Technology (ITEC), Klagenfurt University, Austria, leading the Multimedia Communications group. His current research areas are distributed multimedia systems, multimedia communications, and quality of service. He has received many research grants from national (Austria, Germany) and European funding agencies as well as from industry, is the editor of several books, and has published more than 200 scientific papers on parallel computer architecture, parallel programming, and multimedia communications and adaptation. He is a senior member of the IEEE, member of the ACM, GI (German Informatics Society) and OCG (Austrian Computer Society), and Vice President of the Austrian Science Fund (FWF). 